\title{
Takacs Model of Hysteresis in Mathematical Modeling of Memristors
}

\author{
Stanisa DAUTOVIC, Natasa SAMARDZIC, Anamarija JUHAS \\ Dept. of Power, Electronic and Telecommunication Engineering, Faculty of Technical Sciences, University of Novi Sad, \\ Trg Dositeja Obradovica 6, 21101 Novi Sad, Serbia \\ \{dautovic, nsamardzic, ajuhas\}@uns.ac.rs
}

Submitted July 31, 2019 / Accepted November 10, 2019

\begin{abstract}
In this paper, the mathematical modeling of memristor via Takacs model of hysteresis is presented along with a modification of this model tailored to describe the asymmetric hysteresis loop and first order reversal curves. In particular, it is shown that there is a class of differential equations of the Duhem model of hysteresis where every member of the class could play a role of the state equation of memristor. Within this class of Duhem differential equations, there are two distinct subclasses: one corresponding to the Takacs model and the other one corresponding to the state equations of the memristor model with the Biolek window function of various degrees $p$. These two subclasses have a non-empty intersection, which contains the state equation of the memristor model with the Biolek window function for $p=1$. To demonstrate the proposed approach, three examples are presented.
\end{abstract}

\section{Keywords}

Hysteresis loop, Takacs model, pinched hysteresis loop, memristor

\section{Introduction}

The hysteresis is a nonlinear phenomenon, which emerges in numerous fields, including physics, electronics, materials sciences, biology, mechanics, economy, etc. [1]. It was first observed in ferromagnetic materials, and later in smart materials such as piezoelectric materials, electroactive polymers, magnetostrictive materials, shape memory alloys, etc. [2].

Due to the long history of the subject and the omnipresence of hysteresis phenomena, there exist a considerable number of models and published books on the modeling of hysteresis [3]-[12]. The main advantage of mathematical modeling is in its ability to describe experimental data via analytical expressions, which in turn provides a simplified and more efficient analysis of systems exhibiting hysteretic behavior. There is no model of hysteresis loop (HL) capable to grasp all necessary features of the object to be modeled [12].

Mathematical models of HL usually involve functions such as exponential [13], polynomial [14], [15], and rational [16]-[19], then functions represented via Fourier series [20], Langevin [21], [22], Frolich [19], Brillouin function [21], and modified Brillouin function [23], hyperbolic tangent [24], and arcus tangent function [19], [21], [25]-[28]. Takacs model [29], [11] is based on the linear combination of the hyperbolic tangent and linear function. This model describes hysteresis loop with a small number of parameters and can provide a good fit to experimentally obtained data. Various modifications of Takacs model involving Langevin function, arcus tangent, sine of arcus tangent or Frolich function were considered in [21]. Modifications that involve logistic function, algebraic sigmoidal function or Gompertz function are reported in [30]. Apart from the models based on mathematical functions, hysteresis can be also described by differential equations (e.g. Duhem model [5]).

The appearance of pinched hysteresis loop (PHL) in the voltage-current plane is one of the fingerprints of memristive behavior [31], [32], [33]. A comprehensive overview of fingerprints and the history of the subject are presented in [34]. Odd-symmetric self-crossing PHL is recognized as the signature of ideal memristor [35]. For various degrees of degeneracies, the classification of PHLs on self-crossing and touching has been further refined in [36]. Dependence of the type of PHL on the frequency content of the state variable is analyzed in [37]. Under the assumption that constant charge can be delivered to an ideal memristor within the half-period, the influence of the frequency of sinusoidal excitation on the area of PHL is analyzed in [38]. Apart from memristor, memcapacitor and meminductor, the other nonlinear elements in Chua's table also exhibit PHLs in the appropriate planes, as shown in [39]. Non-memristive elements having PHLs in the voltage-current plane are considered in [40]. Physical interpretation of lobe area (LA) of PHL is discussed in [41]. The computation of LA via the time-domain integration in the voltage-current plane has been studied in [42], [43] and 
[44]. Also, the computation of LA from the memristance vs. state map of the ideal and ideal generic memristors is considered in [45].

A simple integrator-multiplier model of PHL has been introduced in [46]. Theory of Lissajous figures were applied to the creation of PHL and experimentally verified in [47]. A graphical modeling of PHL that exhibits fingerprints of a memristor is presented in [48].

Mathematical model for the major and minor PHLs of memdiode (diode with memory) is proposed in [49] and then modified in [50] by introducing the rate-dependent state variable. In both models, the branches of hysteron are logistic curves, expressed via exponential functions. The simple relation that exists between the exponential and hyperbolic tangent function implies that the branches of logistic hysteron can also be expressed via the tangent hyperbolic function.

Hyperbolic-type memristor with memductance expressed via the hyperbolic tangent function of state variable is proposed in [51]. Although not explicitly reported, HL appears in the memductance-flux plane of this type of memristor. (As usual, by flux/charge we meant the time integral of voltage/current.) Similarly, HL in the memductance-flux plane of $\mathrm{TaO}_{x}$ memristor can be observed in simulations with the model described in [52].

In addition, HL has been reported for spintronic memristor in memristance-flux plane [53], for ferroelectric memristor (along with the so-called reversal curves) in memristance-voltage plane [54], and for quantum point contact memristor in resistance-voltage plane [55].

HLs are measured for spintronic synapses for ANNs in memristance-current plane [56], [57]. Both real and imaginary parts of the admittance of RRAM devices described in [58], [59] exhibit hysteretic behavior with the change of the driving voltage. The coexistence of hysteretic memristive and memcapacitive behavior is analyzed in [60], [61]. Gas discharge lamps are the subclass of memristors having non-crossing PHLs [31] and inverse hysteresis in the flux-charge plane [62].

In this paper, we relate the classical hysteresis theory (Takacs and Duhem models) to the mathematical modeling of memristor. According to our best knowledge, the first usage of the Takacs model of hysteresis in mathematical modeling of memristor is proposed here. We also propose the modified Takacs model, in order to be able to describe asymmetric HL and corresponding first order reversal curves. We also provide two examples of the usage of Takacs model in fitting experimentally obtained data of some of fabricated memristors. Additionally, we show that the differential equation corresponding to the Takacs model belongs to a class of differential equations in the Duhem model of hysteresis. In particular, we show that a class of differential equations in the Duhem model can be used as state equations of memristors. Moreover, we show that the state equation of the memristor model with Biolek window function [63] also belongs to that class of Duhem differential equations. Accordingly, hysteresis appears in the state-charge plane for the current-controlled memristor and it is rate-independent. In the special case when $p=1$ in the Biolek window, we show that the steady state solution of the state equation can be expressed in terms of the Takacs model.

The rest of the paper is organized as follows: Takacs model of HL is briefly described in Sec. 2. In Sec. 3, classical Takacs model is extended to the case of asymmetric HL with the first order reversal curves. In Sec. 4, it is shown that a class of differential equations of Duhem model of hysteresis could be used as the state equation of memristor. It is also shown that the state equation of the memristor model with the Biolek window function, as well as the differential equation corresponding to the Takacs model, both belong to the considered class of differential equations of Duhem model. Three examples, including spintronic memristor [53], ferroelectric memristor [54] and memristor model with the Biolek window function [63] are presented in Sec. 5. Conclusions are provided in Sec. 6.

\section{Takacs Model of Hysteresis}

This section provides a brief description of the Takacs model (also called $T(x)$ model) of HL [11], enabling us to model major and minor HLs, inverse hysteresis, first-order and higher-orders reversal curves, demagnetization spiral, etc.

Takacs model is based on the $T(x)$ function, which is a linear combination of a hyperbolic tangent and a linear function:

$$
T(x)=B_{0} \tanh C_{0} x+A_{0} x .
$$

In order to describe branches of the symmetric HL, the hyperbolic part of $T(x)$ is shifted in horizontal direction (to the right and left by $a_{0}$ ) and in vertical direction (up and down by $b_{1}$ ), see Fig. 1 . The ascending branch $f_{+}$for the increasing values of $x$ is described by

$$
f_{+}(x)=B_{0} \tanh \left(C_{0}\left(x-a_{0}\right)\right)+A_{0} x+b_{1},
$$

whereas the descending branch $f_{-}$for the decreasing values of $x$ is described by

$$
f_{-}(x)=B_{0} \tanh \left(C_{0}\left(x+a_{0}\right)\right)+A_{0} x-b_{1} .
$$

The branches have two common points at the tips of HL (see Fig. 1). Since hyperbolic tangent is an odd function, we can assume, without the loss of generality, that

$$
B_{0}>0 .
$$

For periodic driving signal $x=x(t)$, with zero mean and $-X_{\mathrm{m}} \leq x \leq X_{\mathrm{m}}$, the tips occur at $x= \pm X_{\mathrm{m}}$. Symmetric HL is closed when $f_{+}\left(X_{\mathrm{m}}\right)=f_{-}\left(X_{\mathrm{m}}\right)$. This relation can be solved for $b_{1}$ : 


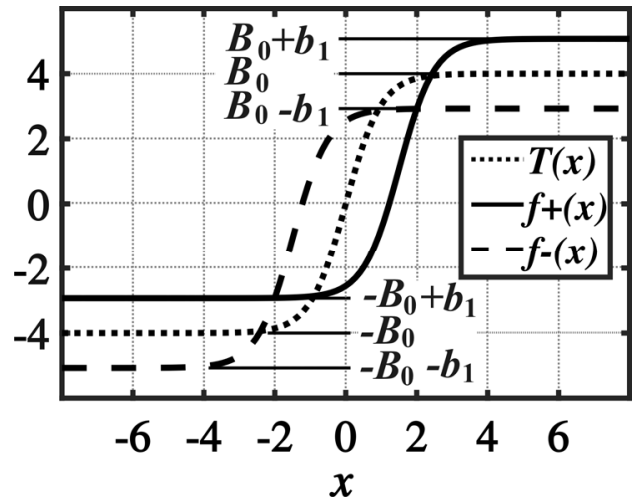

Fig. 1. Examples of $T(x), f_{+}(x)$ and $f_{-}(x)$ functions for $A_{0}=0$, $B_{0}=4, C_{0}=1, a_{0}=1.5, X_{\mathrm{m}}=2$.

$$
b_{1}=\frac{B_{0}}{2}\left[\tanh \left(C_{0}\left(X_{\mathrm{m}}+a_{0}\right)\right)-\tanh \left(C_{0}\left(X_{\mathrm{m}}-a_{0}\right)\right)\right] \text {. }
$$

In the context of magnetic materials, parameter $A_{0}$ in (2) and (3) is related to reversible magnetization, and can be used to "skew" the HL. In what follows, coefficient $A_{0}$ is set to zero. For $A_{0}=0$, the horizontal asymptotes of $f_{+}(x)$ and $f_{-}(x)$ are $\pm B_{0}+b_{1}$ and $\pm B_{0}-b_{1}$, respectively. Notice that HL can be easily shifted up or down by adding the same constant to both $f_{+}$and $f_{-}$.

Setting $A_{0}$ to zero in (2)-(3) and solving the resultant equations for $x$ provide the branches of the inverse hysteresis:

$$
\begin{aligned}
& x_{+}(f)=\frac{1}{C_{0}} \operatorname{artanh} \frac{f-b_{1}}{B_{0}}+a_{0}, \\
& x_{-}(f)=\frac{1}{C_{0}} \operatorname{artanh} \frac{f+b_{1}}{B_{0}}-a_{0}
\end{aligned}
$$

where $\operatorname{artanh}(\cdot)$ denotes the inverse hyperbolic tangent function. Examples of HLs and corresponding inverse HLs are presented in Fig. 2(a) and Fig. 2(b), respectively.

The largest HL that can be achieved in the system or material is by definition the major HL (see e.g. [11]). When the driving signal $x(t)$ is interrupted and reversed, the direction of $f(x)$ is also reversed, see Fig. 3. Corresponding return path is often called the first order reversal curve (FORC) (see e.g. [11]).

Furthermore, when the driving signal is stopped and reversed at $X_{\mathrm{r}}$ on the ascending branch, $-X_{\mathrm{m}}<X_{\mathrm{r}}<X_{\mathrm{m}}$, and
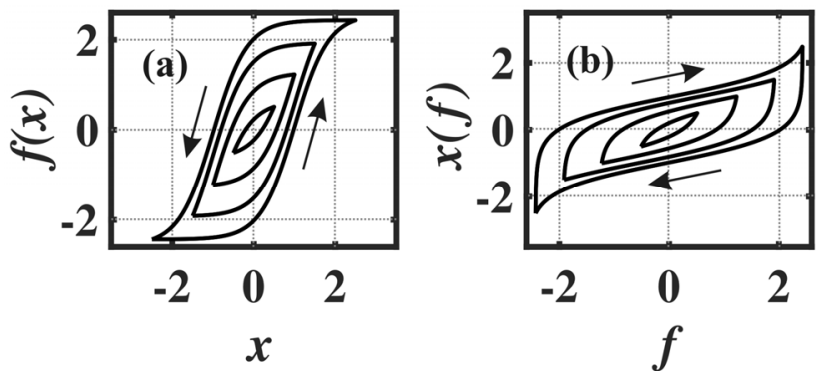

Fig. 2. (a) HLs and (b) corresponding inverse HLs, $B_{0}=2.5$, $a_{0}=1, C_{0}=1.2, X_{\mathrm{m}} \in\{2.5,1.5,1,0.5\}$.
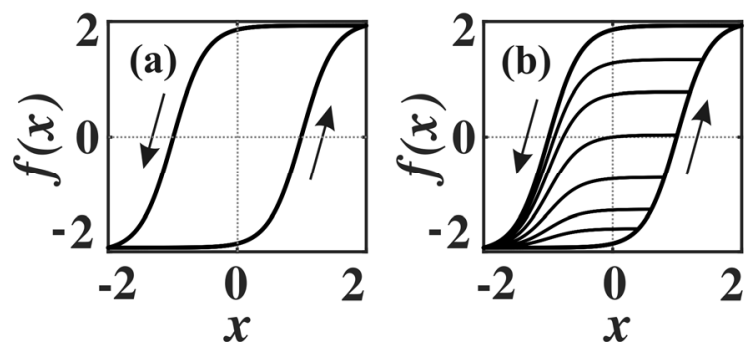

Fig. 3. (a) Major $\mathrm{HL}$ for $B_{0}=2, a_{0}=1, C_{0}=2, X_{\mathrm{m}}=2$ and (b) corresponding FORCs reversing at $X_{\mathrm{r}} \in\{1.4,1.2$, $1,0.8,0.6,0.4\}$.

returned to $-X_{\mathrm{m}}$ (negative saturation), the corresponding return path $f_{\mathrm{r}-}(x)$ can be described by [11]

$$
f_{\mathrm{r}-}(x)=B_{\text {down }} \tanh \left(C_{0}\left(x+a_{0}\right)\right)-c_{\text {down }} .
$$

The ascending branch (2) and down-going return path (8) have two common points: one corresponding to $x=X_{\mathrm{r}}$ and the other to $x=-X_{\mathrm{m}}$. Therefore,

$$
f_{+}\left(X_{\mathrm{r}}\right)=f_{\mathrm{r}-}\left(X_{\mathrm{r}}\right), \quad f_{+}\left(-X_{\mathrm{m}}\right)=f_{\mathrm{r}-}\left(-X_{\mathrm{m}}\right) .
$$

System (9) can be solved for $B_{\text {down }}$ and $c_{\text {down }}$ :

$$
B_{\text {down }}=B_{0} \frac{\beta-\alpha}{\gamma-\delta}, \quad c_{\text {down }}=\left(B_{\text {down }} \delta-B_{0} \alpha\right)-b_{1}
$$

where $b_{1}$ is given by (5) and

$$
\begin{array}{ll}
\alpha=\tanh \left(C_{0}\left(-X_{\mathrm{m}}-a_{0}\right)\right), & \beta=\tanh \left(C_{0}\left(X_{\mathrm{r}}-a_{0}\right)\right), \\
\gamma=\tanh \left(C_{0}\left(X_{\mathrm{r}}+a_{0}\right)\right), & \delta=\tanh \left(C_{0}\left(-X_{\mathrm{m}}+a_{0}\right)\right) .
\end{array}
$$

Dually, when the point of reversal is on the descending branch, the up-going return path $f_{\mathrm{r}^{+}}(x)$ can be described by

$$
f_{\mathrm{r}+}(x)=B_{\mathrm{up}} \tanh \left(C_{0}\left(x-a_{0}\right)\right)+c_{\mathrm{up}} .
$$

The descending branch (3) and up-going return path (12) have two common points: one corresponding to $x=X_{\mathrm{r}}$ and the other to $x=X_{\mathrm{m}}$. By duality, unknown parameters can be obtained from (10)-(11) by replacing $B_{\text {down }}$ with $B_{\text {up }}$, $c_{\text {down }}$ with $c_{\text {up }}, X_{\mathrm{m}}$ with $-X_{\mathrm{m}}$ and $X_{\mathrm{r}}$ with $-X_{\mathrm{r}}$.

\section{Asymmetric HL}

To model asymmetric major HL and corresponding FORCs we provide here a modification of the Takacs model. This type of asymmetric HL and corresponding FORCs are reported in [54] for ferroelectric memristors and in [60] for multilayered metal-oxide structures.

Asymmetric major HL can be modeled via a modified version of the Takacs model by using two sets of parameters: $\left\{C_{0+}, a_{0+}, B_{0+}, b_{1}{ }^{\text {ss }}\right\}$ parameters for the ascending branch and $\left\{C_{0-}, a_{0-}, B_{0-}, b_{1}{ }^{\text {as }}\right\}$ parameters for the descending branch:

$$
\begin{aligned}
& f_{+}^{\text {as }}(x)=B_{0+} \tanh \left(C_{0+}\left(x-a_{0+}\right)\right)+b_{1}^{\text {as }}, \\
& f_{-}^{\text {as }}(x)=B_{0-} \tanh \left(C_{0-}\left(x+a_{0-}\right)\right)-b_{1}^{\text {as }} .
\end{aligned}
$$


For the periodic driving signal $x=x(t)$, with zero dc component and $-X_{\mathrm{m}} \leq x \leq X_{\mathrm{m}}$, the tips of HL occur for $x= \pm X_{\mathrm{m}}$, implying that

$$
f_{+}^{\mathrm{as}}\left(X_{\mathrm{m}}\right)=f_{-}^{\mathrm{as}}\left(X_{\mathrm{m}}\right), \quad f_{+}^{\mathrm{as}}\left(-X_{\mathrm{m}}\right)=f_{-}^{\mathrm{as}}\left(-X_{\mathrm{m}}\right) .
$$

System of equations (15) can be solved for $B_{0-}$ and $b_{1}{ }^{\text {as }}$

$$
B_{0-}=B_{0+} \frac{\beta-\alpha}{\gamma-\delta}, \quad b_{1}^{\mathrm{as}}=\frac{1}{2}\left(B_{0-} \delta-B_{0+} \alpha\right)
$$

where

$$
\begin{aligned}
& \alpha=\tanh \left(C_{0+}\left(-X_{\mathrm{m}}-a_{0+}\right)\right), \beta=\tanh \left(C_{0+}\left(X_{\mathrm{m}}-a_{0+}\right)\right), \\
& \gamma=\tanh \left(C_{0-}\left(X_{\mathrm{m}}+a_{0-}\right)\right), \quad \delta=\tanh \left(C_{0-}\left(-X_{\mathrm{m}}+a_{0-}\right)\right) .
\end{aligned}
$$

When the driving signal is stopped and reversed at $X_{\mathrm{r}}$ on the ascending branch of asymmetric HL, $-X_{\mathrm{m}}<X_{\mathrm{r}}<X_{\mathrm{m}}$, and returned to $-X_{\mathrm{m}}$, the corresponding return path can be described by

$$
f_{\mathrm{r}-}^{\mathrm{as}}(x)=B_{\mathrm{down}}^{\mathrm{as}} \tanh \left(C_{0-}\left(x+a_{0-}\right)\right)-c_{\mathrm{down}}^{\mathrm{as}} .
$$

Since the ascending branch (13) and return path (18) have two common points at $x=-X_{\mathrm{m}}$ and $x=X_{\mathrm{r}}$, it follows that

$$
f_{+}^{\text {as }}\left(X_{\mathrm{r}}\right)=f_{\mathrm{r}-}^{\text {as }}\left(X_{\mathrm{r}}\right), \quad f_{+}^{\text {as }}\left(-X_{\mathrm{m}}\right)=f_{\mathrm{r}-}^{\text {as }}\left(-X_{\mathrm{m}}\right) .
$$

Substitution of (13) and (18) into (19) provides the system of equations, which can be solved for $B_{\text {down }}^{\text {as }}$ and $c_{\text {down }}^{\text {as }}$,

$$
B_{\text {down }}^{\text {as }}=B_{0+} \frac{\beta_{+}-\alpha}{\gamma_{-}-\delta}, \quad c_{\text {down }}^{\text {as }}=\left(B_{\text {down }}^{\text {as }} \delta-B_{0+} \alpha\right)-b_{1}^{\text {as }}
$$

where $b_{1}$ as is given by (16), $\alpha$ and $\delta$ by (17), and

$$
\beta_{+}=\tanh \left(C_{0+}\left(X_{\mathrm{r}}-a_{0+}\right)\right), \gamma_{-}=\tanh \left(C_{0_{-}}\left(X_{\mathrm{r}}+a_{0-}\right)\right) \text {. }
$$

An example of asymmetric major HL along with FORCs is presented in Fig. 4.

Dually, when the point of reversal is on the descending branch of asymmetric HL, the up-going return path $f_{\mathrm{r}+}(x)$ can be described by

$$
f_{\mathrm{r}+}^{\mathrm{as}}(x)=B_{\mathrm{up}}^{\mathrm{as}} \tanh \left(C_{0+}\left(x-a_{0+}\right)\right)+c_{\mathrm{up}}^{\text {as }} .
$$

Notice that the descending branch (14) and return path (22) have two common points corresponding to $x=X_{\mathrm{m}}$ and $x=X_{\mathrm{r}}$, i.e. $f_{-}^{\text {as }}\left(X_{\mathrm{m}}\right)=f_{\mathrm{r}^{+}}$as $\left(X_{\mathrm{m}}\right)$ and $f_{-}^{\text {as }}\left(X_{\mathrm{r}}\right)=f_{\mathrm{r}^{+}}$as $\left(X_{\mathrm{r}}\right)$.
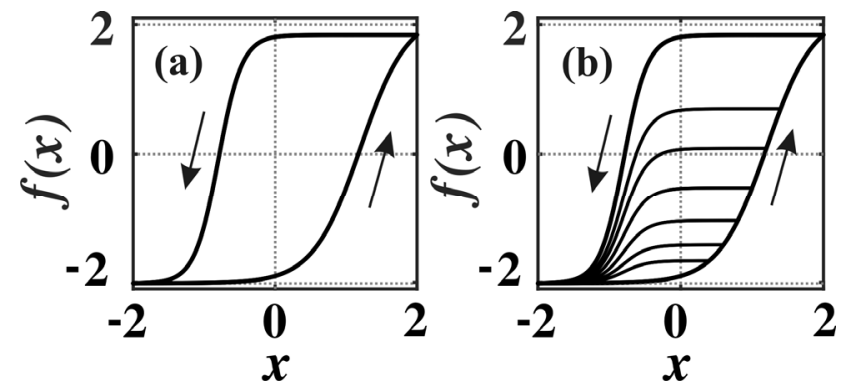

Fig. 4. (a) Asymmetric major HL for $B_{0+}=2.1, C_{0+}=1.5$, $a_{0+}=1.2, C_{0-}=3, a_{0-}=0.8, X_{\mathrm{m}}=2$ and (b) FORCs reversing at $X_{\mathrm{r}} \in\{1.4,1.2,1,0.8,0.6,0.4\}$.
From these relations, the unknown parameters $B_{\text {up }}^{\text {as }}$ and $c_{\text {up }}^{\text {as }}$ can be easily determined.

\section{State Equations and Hysteresis}

In this section, we show that a class of differential equations of the Duhem model of hysteresis could play a role of state equations in the mathematical models of memristors. Since the Duhem model describes rate independent hysteresis, it follows that memristors with state equations of Duhem type exhibit rate-independent hysteresis in the state-charge (state-flux) plane for current-controlled (voltage-controlled) memristor. We also show that the state equation for memristor model with the Biolek window function belongs to the considered class of differential equations of Duhem model. Additionally, we demonstrate that the differential equation that corresponds to the Takacs model also belongs to the same class.

Let us consider a class of differential equations of the Duhem model of hysteresis in $x-U$ plane. The corresponding equation is of the form

$$
\frac{\mathrm{d} x}{\mathrm{~d} t}=\max (0, u) g(x)+\min (0, u) h(x)
$$

where $x$ stands for a variable, $x(0)=x_{0}$ is the initial condition, and $u$ is the first derivative of $U$ with respect to time, i.e.

$$
\frac{\mathrm{d} U}{\mathrm{~d} t}=u
$$

$\max / \mathrm{min}$ denotes the maximum/minimum function, and $g(x)$ and $h(x)$ are continuous functions. We consider a class of Duhem equations (23) in which $g$ and $h$ are functions of the variable $x$ only, and hence independent of $U$. Equations (23), (24) represent the Duhem model of hysteresis, which is rate-independent, e.g. [5].

Both formation and disruption of filamentary like conductive channels across the insulating film is described by the differential equation of type (23) in [49]. In the model described by Equation (12) of [49], $U$ corresponds to voltage and $u$ to the first derivative of voltage, respectively.

It is easy to show that (23) can be rewritten as

$$
\frac{\mathrm{d} x}{\mathrm{~d} t}=[H(u) g(x)+H(-u) h(x)] u
$$

where $H(\cdot)$ is the Heaviside step function: $H(u)=1$ for $u \geq 0$, and $H(u)=0$ for $u<0$. According to the Duhem model, hysteresis will appear in the $x-U$ plane, where

$$
U=\int u \mathrm{~d} t
$$

Additionally, (25) can be rewritten as

$$
\frac{\mathrm{d} x}{\mathrm{~d} t}=w(x, u) u
$$


where

$$
w(x, u)=H(u) g(x)+H(-u) h(x) .
$$

In the context of memristors, (27) is state equation in terms of $x$ (state variable) and $u$ (the first derivative of $U$ ). In the same context, $u$ is the driving voltage or driving current. According to the Duhem model, the solution of (27)-(28) exhibits the rate-independent hysteresis in the $x$-charge plane for current-controlled memristors or $x$ - flux plane for voltage-controlled memristors.

Particularly, substitution of

$$
g(x)=k\left(1-x^{2 p}\right), \quad h(x)=k\left[1-(1-x)^{2 p}\right]
$$

where $k$ is constant and $p$ positive integer, along with $u=i$ into (27), (28) leads to

$$
\frac{\mathrm{d} x}{\mathrm{~d} t}=k w_{2 p}(x, i) i
$$

where

$$
w_{2 p}(x, i)=H(i)\left(1-x^{2 p}\right)+H(-i)\left[1-(1-x)^{2 p}\right] .
$$

It is easy to show that $w_{2 p}(x, i)$ for $0<x<1$ coincides with the Biolek window function introduced in [63]. Therefore, (30) is the state equation for the current-controlled memristor with the Biolek window function. According to the Duhem model, hysteresis appears in the state-charge plane. A case study corresponding to the model of the currentcontrolled memristor with the Biolek window function is presented in Sec. 5.3.

In what follows we derive the differential equation that corresponds to the Takacs model of hysteresis. We show that this Takacs differential equation is of type (27) and therefore can also be used in the modeling of memristors. For a particular choice of parameters, the Takacs differential equation coincides with the state equation of the Biolek model for $p=1$, as it is presented in Sec. 5.3.

Let us assume that the state variable with hysteresis, shifted in the horizontal direction by $d_{0}$ and in the vertical direction by $X_{0}$, is described by

$$
x= \begin{cases}B_{0} \tanh \left(C_{0}\left(U-a_{0}+d_{0}\right)\right)+b_{1}+X_{0}, & u>0, \\ B_{0} \tanh \left(C_{0}\left(U+a_{0}+d_{0}\right)\right)-b_{1}+X_{0}, & u<0 .\end{cases}
$$

Recall that it can be assumed, without loss of generality, that $B_{0}>0$. Since the co-domain of the tangent hyperbolic function of finite argument is an open interval $(-1,1)$, it follows from (32) that

$$
\begin{array}{ll}
X_{0}-B_{0}+b_{1}<x<X_{0}+B_{0}+b_{1}, & u>0, \\
X_{0}-B_{0}-b_{1}<x<X_{0}+B_{0}-b_{1}, & u<0 .
\end{array}
$$

Solving (33) we obtain the following range for the state variable with hysteresis described by (32),

$$
X_{0}-\left[B_{0}-\left|b_{1}\right|\right]<x<X_{0}+\left[B_{0}-\left|b_{1}\right|\right],
$$

providing that

$$
B_{0}>\left|b_{1}\right| \text {. }
$$

Taking into account that the first derivative of hyperbolic tangent function satisfies the following identity

$$
\frac{\mathrm{d}}{\mathrm{d} z} \tanh (z)=1-\tanh ^{2}(z)
$$

the first derivative of (32) with respect to the time can be expressed as

$$
\frac{\mathrm{d} x}{\mathrm{~d} t}=u \frac{C_{0}}{B_{0}} \begin{cases}B_{0}^{2}-\left(x-b_{1}-X_{0}\right)^{2}, & u>0, \\ B_{0}^{2}-\left(x+b_{1}-X_{0}\right)^{2}, & u<0 .\end{cases}
$$

This expression can be rewritten in the form (27)-(28) providing that

$$
\begin{aligned}
& g(x)=\frac{C_{0}}{B_{0}}\left[B_{0}^{2}-\left(x-b_{1}-X_{0}\right)^{2}\right], \\
& h(x)=\frac{C_{0}}{B_{0}}\left[B_{0}^{2}-\left(x+b_{1}-X_{0}\right)^{2}\right] .
\end{aligned}
$$

Expression (32) describes both the accommodation, as the transient part of the process, and closed HL as the corresponding limit cycle. Thus, the steady state solution of (37), as a limit cycle, is independent of initial conditions [64].

For the prescribed $B_{0}, C_{0}, b_{1}$ and $X_{0}$, the remaining parameters $a_{0}$ and $d_{0}$ of closed HL (32) can be determined from the coordinates of the tips $\left(0, x_{\min }\right)$ and $\left(U_{\max }, x_{\max }\right)$, where $\min (U)=0$ and $\max (U)=U_{\max }$. Appendix contains the full account of the arguments for the derivation of the following relations

$$
\begin{gathered}
x_{\text {max }}+x_{\min }=2 X_{0}, \\
d_{0}-a_{0}=\frac{1}{C_{0}} \operatorname{artanh} \frac{X_{0}-x_{\max }-b_{1}}{B_{0}}, \\
d_{0}+a_{0}=-\frac{1}{C_{0}} \operatorname{artanh} \frac{x_{\max }-X_{0}-b_{1}}{B_{0}}, \\
x_{\max }=\frac{B_{0}-\sqrt{B_{0}^{2}\left(1-\alpha^{2}\right)+b_{1}^{2} \alpha^{2}}}{\alpha}
\end{gathered}
$$

where $\alpha=\tanh \left(C_{0} U_{\max }\right)$. The substitution of (42) into (40), (41), followed by the substitution of resulting relations into
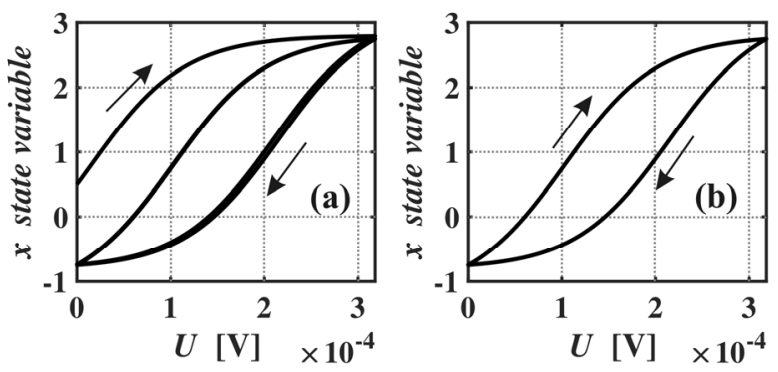

Fig. 5. (a) Solution of (37) obtained numerically and (b) HL (32) for $x_{0}=0.5, u(t)=U_{\mathrm{m}} \sin (2 \pi f t), U_{\mathrm{m}}=1$, $f=1 \mathrm{~Hz}, B_{0}=2, b_{1}=-0.2, X_{0}=1$, and $C_{0}=10^{4}$. 
(32), provides the closed-form expression for the steady state solution of differential equation corresponding to the Takacs model. This result is also confirmed by the numerical simulations. The solution of the differential equation (37) for the prescribed initial condition, which includes transients and steady state, is presented in Fig. 5(a), while the steady state solution only is presented in Fig. 5(b).

\section{Examples of Modeling HL}

In this section, three examples of modeling HL are presented. Section 5.1 is related to spintronic memristor [53], Section 5.2 to ferroelectric memristor [54], and Section 5.3 to the model of memristor with the Biolek window function [63].

\subsection{Spintronic Memristor}

HL for spintronic memristor in the memristance-flux plane is reported in [53]. The branches of HL are described by

$$
R^{ \pm}(\Phi)=R_{\mathrm{H}}-\frac{R_{\mathrm{H}}-R_{\mathrm{L}}}{1+\exp \left[\left(\Phi_{\mathrm{S}}^{ \pm}-\Phi\right) / \delta^{ \pm}\right]}
$$

where $R^{+}$is the branch corresponding to positive voltage (increasing flux), $R^{-}$is the branch corresponding to negative voltage (decreasing flux), and flux is in the range $\Phi \in[0,84]$ Vs. In the anti-parallel magnetic state, high and low resistances are $R_{\mathrm{H}}=375.9 \Omega$ and $R_{\mathrm{L}}=362.5 \Omega$, respectively, whereas in the parallel magnetic state, they are $R_{\mathrm{H}}=189.6 \Omega$ and $R_{\mathrm{L}}=178.9 \Omega$, respectively. The remaining parameters are obtained by fitting [53]: $\Phi_{\mathrm{S}}{ }^{+}=19.5 \mathrm{Vs}$, $\delta^{+}=4.3 \mathrm{~V}, \Phi_{\mathrm{S}}^{-}=52.8 \mathrm{Vs}$, and $\delta^{-}=5.3 \mathrm{~V}$.

Using the identity

$$
\tanh (x)=1-\frac{2}{\mathrm{e}^{2 x}+1},
$$

branches of (43) can be rewritten as

$$
R^{ \pm}(\Phi)=\frac{R_{\mathrm{H}}-R_{\mathrm{L}}}{2} \tanh \frac{\Phi_{\mathrm{S}}^{ \pm}-\Phi}{2 \delta^{ \pm}}+\frac{R_{\mathrm{H}}+R_{\mathrm{L}}}{2} .
$$

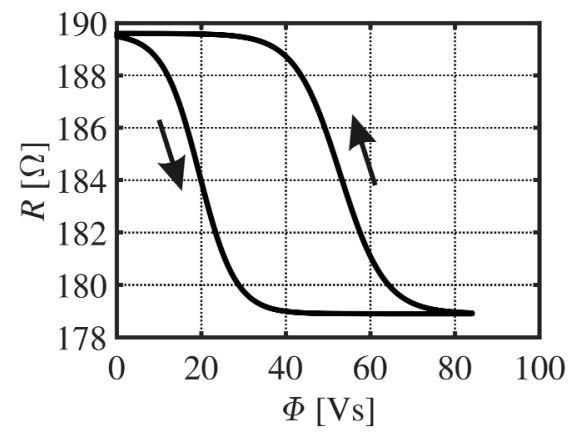

Fig. 6. $\mathrm{HL}$ of spintronic memristor, $B_{0+}=B_{0-}=5.35 \Omega$, $a_{0}=-16.65 \mathrm{Vs}, \quad d_{0}=36.15 \mathrm{Vs}, \quad C_{0+}=-0.1163 \mathrm{~V}^{-1}$, $C_{0-}=-0.0943 \mathrm{~V}^{-1}, b_{1}=0$, and $R_{0}=184.25 \Omega$.
On the other hand, asymmetric HL (Sec. 3), shifted horizontally by $d_{0}$ and vertically by $R_{0}$, can be described by

$$
\begin{aligned}
& R_{+}=B_{0+} \tanh \left(C_{0+}\left(\Phi-a_{0}-d_{0}\right)\right)+b_{1}+R_{0}, \\
& R_{-}=B_{0-} \tanh \left(C_{0-}\left(\Phi+a_{0}-d_{0}\right)\right)-b_{1}+R_{0} .
\end{aligned}
$$

Comparison of (45) and (46) provides the following parameter values:

$$
\begin{array}{ll}
B_{0+}=B_{0-}=\frac{1}{2}\left(R_{\mathrm{H}}-R_{\mathrm{L}}\right), & \\
C_{0+}=-\frac{1}{2 \delta^{+}}, & C_{0-}=-\frac{1}{2 \delta^{-}}, \\
a_{0}=\frac{1}{2}\left(\Phi_{\mathrm{S}}^{+}-\Phi_{\mathrm{S}}^{-}\right), & d_{0}=\frac{1}{2}\left(\Phi_{\mathrm{S}}^{+}+\Phi_{\mathrm{S}}^{-}\right), \\
b_{1}=0 \Omega, & R_{0}=\frac{1}{2}\left(R_{\mathrm{H}}+R_{\mathrm{L}}\right) .
\end{array}
$$

Thus, model (43) can be rewritten in terms of modified Takacs model proposed in Sec. 3. In the case of classical hysteresis, the maximum/minimum input corresponds to the maximum/minimum output. In this example, the maximum resistance corresponds to the minimum flux and vice versa. This can be easily handled with both Takacs model and modified Takacs model by using negative values for parameters $C_{0+}$ and $C_{0-}$. HL described by (46)-(47) is presented in Fig. 6.

\subsection{Ferroelectric Memristor}

Let us consider voltage-controlled ferroelectric memristor, with two orders of magnitude of OFF-to-ON resistance ratio and tunable intermediate states, as presented in [54]. The result of measurements in memristancevoltage plane is depicted in Fig. 7(a).

In this case study, we model the FORCs related to the multilevel resistance states displayed in Fig. 7(a). The FORCs begin on the ascending branch and end at the lower left corner, where memristance is equal to $R_{\mathrm{ON}}$. We estimate $R_{\mathrm{ON}} \approx 0.18 \mathrm{M} \Omega$. The minimum voltage $V_{\min } \approx-6 \mathrm{~V}$
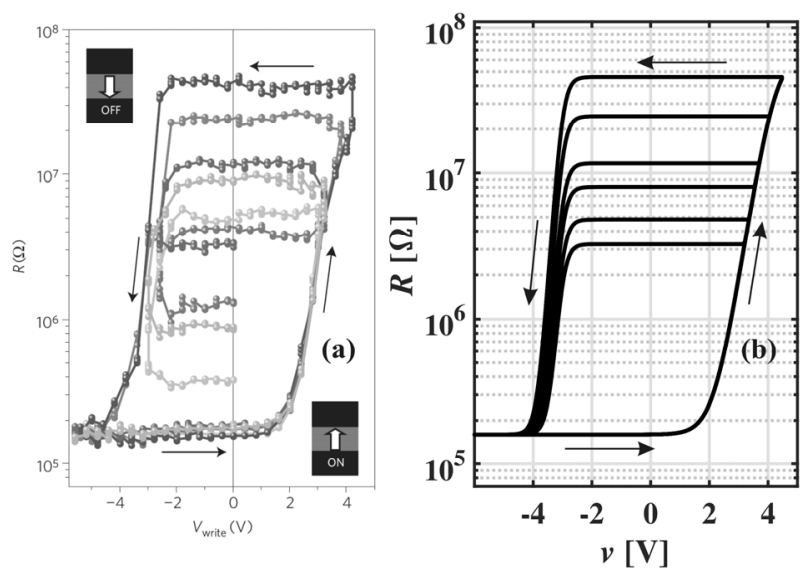

Fig. 7. (a) $R-V_{\text {write }}$ of ferroelectric memristor (reprinted by permission from Ref. [54], Copyright (2012) Springer Nature, Nat. Mater.) and (b) model of FORCs. 
and the maximum voltage $V_{\max } \approx 4.5 \mathrm{~V}$, which can be read on the horizontal axis in Fig. 7(a), imply that the descending branch of major $\mathrm{HL}$ is missing. Thus, we adopt $V_{\mathrm{m}}=6 \mathrm{~V}$, and estimate that the set of reversal points on the ascending branch is $V_{\mathrm{r}} \in\{4.5,4.05,3.7,3.55,3.35,3.2\} \mathrm{V}$. Likewise, we assume that $R_{\mathrm{OFF}} \approx 68 \mathrm{M} \Omega$.

Here, we use the model of asymmetric HL with corresponding FORCs as described in Sec. 3. Additionally, we shift the model vertically by $R_{0}$. The ascending branch for increasing voltage can be written as

$$
R_{+}=B_{0+} \tanh \left(C_{0+}\left(v-a_{0+}\right)\right)+b_{1}^{\text {as }}+R_{0},
$$

and the descending branch as

$$
R_{-}=B_{0-} \tanh \left(C_{0-}\left(v+a_{0-}\right)\right)-b_{1}^{\text {as }}+R_{0} .
$$

Additionally, FORCs for decreasing voltage have the form

$$
R_{\mathrm{r}-}=B_{\text {down }}^{\text {as }} \tanh \left(C_{0-}\left(v+a_{0-}\right)\right)-c_{\text {down }}^{\text {as }}+R_{0} .
$$

Parameter $R_{0}$ is set to $\left(R_{\mathrm{OFF}}+R_{\mathrm{ON}}\right) / 2$, i.e. $R_{0}=34.08 \mathrm{M} \Omega$, while $B_{0+}$ and $B_{0-}$ are calculated as (see (16)-(17))

$$
\begin{aligned}
& B_{0+}=\left(R_{\mathrm{OFF}}-R_{\mathrm{ON}}\right) \frac{\gamma-\delta}{\gamma-\delta+\beta-\alpha}=34.026 \mathrm{M} \Omega, \\
& B_{0-}=\left(R_{\mathrm{OFF}}-R_{\mathrm{ON}}\right) \frac{\beta-\alpha}{\gamma-\delta+\beta-\alpha}=33.814 \mathrm{M} \Omega .
\end{aligned}
$$

Parameters $C_{0+}, a_{0+}, C_{0-}$, and $a_{0-}$ are used for fitting the major HL. Their values are: $C_{0+}=1.45 \mathrm{~V}^{-1}, a_{0+}=4.25 \mathrm{~V}$, $C_{0-}=3.3 \mathrm{~V}^{-1}$, and $a_{0-}=3 \mathrm{~V}$. Parameter $b_{1}{ }^{\text {as }}$ can be obtained by substituting $X_{\mathrm{m}}=V_{\mathrm{m}}$ into (16). Furthermore, parameters $B_{\text {down }}^{\text {as }}$ and $c_{\text {down }}^{\text {as }}$ can be calculated from (20) using that $X_{\mathrm{m}}=V_{\mathrm{m}}$ and $X_{\mathrm{r}}=V_{\mathrm{r}}$. The result of modeling is shown in Fig. 7(b).

\subsection{Memristor Model with Biolek Window}

As presented in Sec. 4, the solution of the state equation of the current-controlled memristor with the Biolek window function exhibits rate-independent hysteresis in the state-charge plane.

In this subsection, we provide parameters for which the differential equation of the Takacs model (37) is reduced to the state equation with the Biolek window for $p=1$. Since the steady state solution of (37) is given by (32), it immediately follows that the same set of parameters provides the closed-form expression for the steady state solution of the state equation with the Biolek window for $p=1$. Additionally, we demonstrate that, for the steady state solution of state equation with the Biolek window for $p \geq 1$, the maximum value $x_{\max }$ and the minimum value $x_{\min }$ of the state variable satisfy the relation $x_{\max }+x_{\min }=1$. This relation can be used to estimate transient time as well as a numerical error in the course of finding the steady state solution.

Since the memristance of the model with the Biolek window is represented as a linear function of state variable,

$$
R(x)=R_{\text {off }}-\left(R_{\text {off }}-R_{\text {on }}\right) x,
$$

it is easy to validate that the existence of the hysteresis in the state-charge plane implies the existence of hysteresis in the memristance-charge plane. State variable vs. charge and memristance vs. charge for memristor model with the Biolek window for $p \in\{1,2,10\}$, are presented in Fig. 8(a) and Fig. 8(b), respectively. Simulations were performed using the Runge-Kutta-Fehlberg 4(5) method.

Notice that it has been recently proved that the memristor model with the Biolek window is characterized by a single stable fixed point [65], [66], meaning that the model is globally asymptotically stable.

Fig. 9 presents HLs in the memristance-charge plane and corresponding PHLs for three different driving oddsymmetric periodic current waveforms. For prescribed $Q_{\max }=\max (Q)$ and given frequency $f$, the corresponding amplitudes for sinusoidal, triangular and rectangular currents are equal to $Q_{\max } \pi f, Q_{\max } 4 f$, and $Q_{\max } 2 f$, respectively. HLs in the memristance-charge plane in Fig. 9(a) coincide for all three waveforms, which is in accordance with the fact that the Duhem model describes the rate-independent hysteresis.

By comparison of (37) with (30), (31), we conclude that the substitution of $B_{0}=1, C_{0}=k, b_{1}=-1 / 2, X_{0}=1 / 2$ and $u=i$ into (37) provides the state equation with the Biolek window for $p=1$ :

$$
\frac{\mathrm{d} x}{\mathrm{~d} t}=k i\left\{\begin{array}{cc}
1-x^{2}, & i>0, \\
1-(x-1)^{2}, & i<0 .
\end{array}\right.
$$
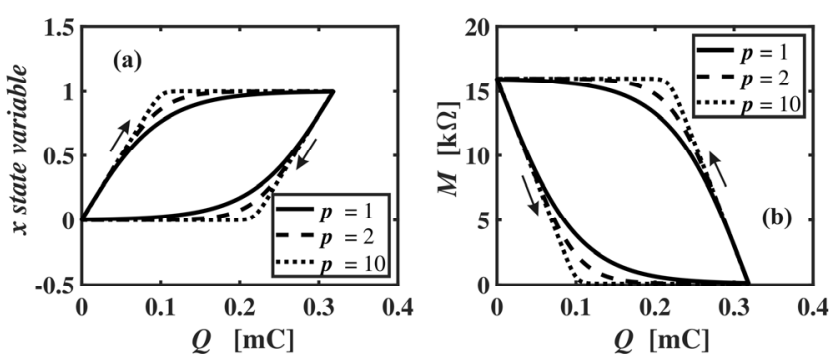

Fig. 8. Steady state solutions of state equation for the currentcontrolled memristor with the Biolek window for $p \in\{1,2,10\}, k=10^{4}, i(t)=I_{\mathrm{m}} \sin (2 \pi f t), \quad I_{\mathrm{m}}=1 \mathrm{~mA}$, and $f=1 \mathrm{~Hz}$, (a) state variable vs. charge and (b) memristance vs. charge.
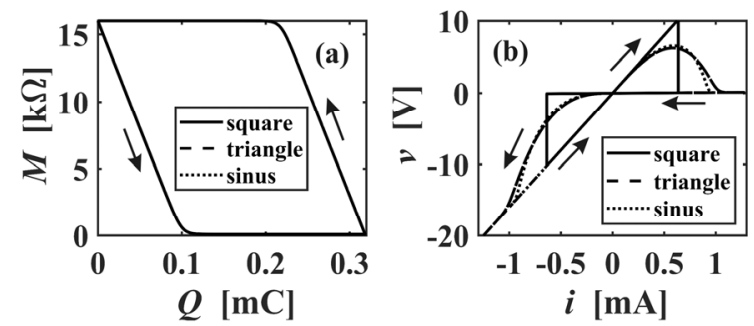

Fig. 9. Model with the Biolek window for $p=10, k=10^{4}$, $R_{\text {off }}=16 \mathrm{k} \Omega, R_{\text {on }}=100 \Omega$, and three driving currents: sinusoidal, triangular and rectangular with the same $Q_{\max }=1 / \pi \mathrm{mC}$ and $f=1 \mathrm{~Hz}$. (a) HLs in the memristance-charge plane and (b) corresponding PHLs in the voltage-current plane. 
Thus, the Takacs model of hysteresis is closely related to the model of memristor with the Biolek window function for $p=1$.

Furthermore, the substitution of $B_{0}=1, C_{0}=k$, $b_{1}=-1 / 2, X_{0}=1 / 2, u=i$ and $U=Q$ into (32) yields

$$
x=\left\{\begin{array}{cc}
\tanh \left(k\left(Q-a_{0}+d_{0}\right)\right), & i>0, \\
\tanh \left(k\left(Q+a_{0}+d_{0}\right)\right)+1, & i<0 .
\end{array}\right.
$$

Substitution of the same values of parameters along with $U_{\max }=Q_{\max }$ into (39)-(42) yields

$$
\begin{gathered}
x_{\text {max }}+x_{\text {min }}=1, \\
d_{0}-a_{0}=\frac{1}{k} \operatorname{artanh}\left(1-x_{\max }\right), \\
d_{0}+a_{0}=-\frac{1}{k} \operatorname{artanh}\left(x_{\max }\right), \\
x_{\max }=\frac{2+\alpha-\sqrt{4-3 \alpha^{2}}}{2 \alpha}
\end{gathered}
$$

where $\alpha=\tanh \left(k Q_{\max }\right)$. Substitution of (56)-(58) into (54) provides the steady state solution of the state equation with the Biolek window for $p=1$.

As an example, Fig. 10 presents both the numerical solution with transients (dotted line) and the steady state solution obtained analytically (solid line), for two different amplitudes of sinusoidal driving current.

The solution of the state equation with the Biolek window for $p>1$, which reads

$$
\frac{\mathrm{d} x}{\mathrm{~d} t}=k i\left\{\begin{array}{cc}
1-x^{2 p}, & i>0 \\
1-(1-x)^{2 p}, & i<0
\end{array}\right.
$$

cannot be expressed in the form (32). It is easy to observe that (59) can be rewritten as

$$
i \mathrm{~d} t=\frac{1}{k}\left\{\begin{array}{cc}
\frac{\mathrm{d} x}{1-x^{2 p}}, & i>0, \\
\frac{\mathrm{d} x}{1-(1-x)^{2 p}}, & i<0 .
\end{array}\right.
$$

$$
\text { Let } \quad W_{2 p}(x)=\int \frac{\mathrm{d} x}{1-x^{2 p}} .
$$

$W_{2 p}(x)$ can be expressed in terms of Gauss Hypergeometric function, e.g. [67], as

$$
W_{2 p}(x)=x \cdot{ }_{2} F_{1}\left[\frac{1}{2 p}, 1,1+\frac{1}{2 p} ; x^{2 p}\right] .
$$

Since (60) is a separable equation, taking into account (62), (61) and $Q=\int_{0}^{t} i \mathrm{~d} \tau$, the solution of (60) can be expressed as

$$
Q=\frac{1}{k}\left\{\begin{array}{cc}
W_{2 p}(x)+a_{0}-d_{0}, & i>0, \\
-W_{2 p}(1-x)-a_{0}-d_{0}, & i<0,
\end{array}\right.
$$
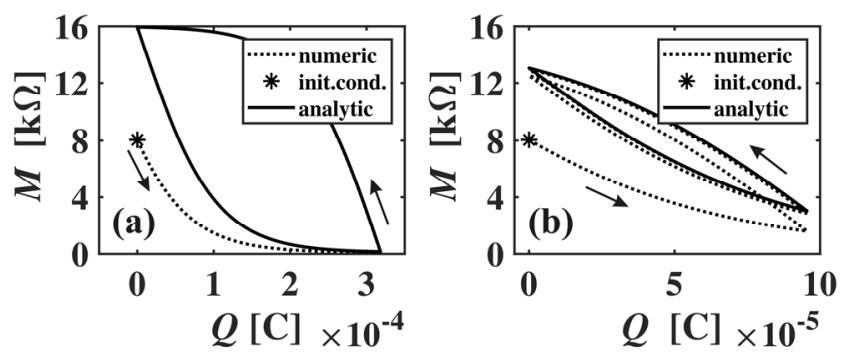

Fig. 10. Steady state solution (solid line) vs. numerical (dotted line) solution in the memristance-charge plane for the model with the Biolek window for $p=1, k=10^{4}$, $R_{\text {off }}=16 \mathrm{k} \Omega, R_{\text {on }}=100 \Omega, x_{0}=0.5, i(t)=I_{\mathrm{m}} \sin (2 \pi f t)$, $f=1 \mathrm{~Hz}$ : (a) $I_{\mathrm{m}}=1 \mathrm{~mA}$, and (b) $I_{\mathrm{m}}=0.3 \mathrm{~mA}$.

where $d_{0}$ and $a_{0}$ are constants. Since the coordinates of the tips of HL are $\left(Q_{\max }, x_{\max }\right)$ and $\left(0, x_{\min }\right)$, the corresponding set of equations can be obtained from (66)-(69) (see Appendix), by inserting $B_{0}=1, C_{0}=k, b_{1}=-1 / 2, X_{0}=1 / 2$, $U_{\max }=Q_{\max }$, and then replacing $\operatorname{artanh}(x)$ with $W_{2 p}(x)$ and $\operatorname{artanh}(1-x)$ with $W_{2 p}(1-x)$. The same steps as in Appendix provide

$$
W_{2 p}\left(x_{\max }\right)+W_{2 p}\left(1-x_{\max }\right)=W_{2 p}\left(1-x_{\min }\right)+W_{2 p}\left(x_{\min }\right) .
$$

Taking into account that the first derivative of the auxiliary function $W_{2 p}^{\text {aux }}(x)=W_{2 p}(x)+W_{2 p}(1-x)$ equals to

$$
\frac{\mathrm{d}}{\mathrm{d} x} W_{2 p}^{\mathrm{aux}}(x)=\frac{1}{1-x^{2 p}}-\frac{1}{1-(1-x)^{2 p}},
$$

and using the same arguments as in Appendix, it can be demonstrated that (55) holds for the steady state solution and $p>1$ in the Biolek window, as well.

Since the Gauss Hypergeometric function ${ }_{2} F_{1}$ is expressed via an infinite series, its simulation is usually slow and it is recommendable to use an efficient algorithm for solving differential equations, instead. In such case, relation (55) can be used to verify whether the steady state solution is reached in numerical simulations and/or to estimate the numerical errors of the solutions.

\section{Conclusion}

According to our best knowledge, this paper provides the first usage of the Takacs model of hysteresis in the mathematical modeling of memristor. A modified version of the Takacs model tailored to describe asymmetric HL and corresponding FORCs, which are observed in some experiments, is also proposed.

In particular, it is presented that a class of differential equations of the Duhem model of hysteresis coincides with a class of state equations of memristors. In this context, hysteresis appears in the state-charge (state-flux) plane for current-controlled (voltage-controlled) memristors. It is also confirmed that the state equation of the memristor model with the Biolek window function, as well as the differential equation that corresponds to the Takacs model, both belong to the class of differential equations related to 
the Duhem model. Since the Duhem model describes rateindependent hysteresis, it follows that hysteresis in the appropriate planes of memristors is also rate-independent.

Notice that in the Takacs model, the sigmoidal function other than the tangent hyperbolic function is also possible.

We believe that the idea behind this paper can be extended to the modeling of memcapacitor, meminductor and other mem-devices that exhibit HLs in the appropriate planes.

\section{Appendix}

Here we provide the derivation of expressions (39)-(42) presented in Sec. 4.

Substitution of the coordinates of the tips $\left(U_{\max }, x_{\max }\right)$ and $\left(0, x_{\min }\right)$, into (32) yields the following system of equations:

$$
\begin{gathered}
C_{0}\left(d_{0}-a_{0}\right)=\operatorname{artanh} \frac{x_{\min }-X_{0}-b_{1}}{B_{0}}, \\
C_{0}\left(d_{0}+a_{0}\right)=-\operatorname{artanh} \frac{X_{0}-x_{\min }-b_{1}}{B_{0}}, \\
C_{0}\left(U_{\max }+d_{0}-a_{0}\right)=\operatorname{artanh} \frac{x_{\text {max }}-X_{0}-b_{1}}{B_{0}}, \\
C_{0}\left(U_{\max }+d_{0}+a_{0}\right)=-\operatorname{artanh} \frac{X_{0}-x_{\max }-b_{1}}{B_{0}} .
\end{gathered}
$$

Insertion of (66) into (68) and (67) into (69) leads to

$$
\begin{aligned}
& C_{0} U_{\text {max }}=\operatorname{artanh} \frac{x_{\max }-X_{0}-b_{1}}{B_{0}}-\operatorname{artanh} \frac{x_{\min }-X_{0}-b_{1}}{B_{0}}, \\
& C_{0} U_{\text {max }}=\operatorname{artanh} \frac{X_{0}-x_{\min }-b_{1}}{B_{0}}-\operatorname{artanh} \frac{X_{0}-x_{\max }-b_{1}}{B_{0}} .
\end{aligned}
$$

Further, insertion of (70) into (71) yields

$$
\begin{aligned}
& \operatorname{artanh} \frac{x_{\max }-X_{0}-b_{1}}{B_{0}}+\operatorname{artanh} \frac{X_{0}-x_{\max }-b_{1}}{B_{0}} \\
& =\operatorname{artanh} \frac{X_{0}-x_{\min }-b_{1}}{B_{0}}+\operatorname{artanh} \frac{x_{\min }-X_{0}-b_{1}}{B_{0}} .
\end{aligned}
$$

In order to demonstrate that (39) is the only solution of (72) let us introduce an auxiliary function

$$
W_{\mathrm{T}}^{\text {aux }}(x)=\operatorname{artanh} \frac{x-X_{0}-b_{1}}{B_{0}}+\operatorname{artanh} \frac{X_{0}-x-b_{1}}{B_{0}} .
$$

The first derivative of $W_{T}^{\text {aux }}(x)$ with respect to $x$ reads

$$
\frac{\mathrm{d}}{\mathrm{d} x} W_{\mathrm{T}}^{\text {aux }}(x)=\frac{B_{0}}{B_{0}^{2}-\left(x-X_{0}-b_{1}\right)^{2}}-\frac{B_{0}}{B_{0}^{2}-\left(X_{0}-x-b_{1}\right)^{2}} .
$$

It is easy to observe that the right hand side of (74) is equal to zero at $x=X_{0}$. Furthermore, the sign of the right hand side is the same as the sign of $b_{1}$ for $x<X_{0}$ and opposite for $X_{0}<x$. Therefore, for $b_{1}>0\left(b_{1}<0\right) W_{\mathrm{T}}^{\text {aux }}(x)$ is monotonically increasing (decreasing) for $x<X_{0}$ and monotonically decreasing (increasing) for $X_{0}<x$. These facts further imply that $W_{\mathrm{T}}^{\text {aux }}(x)$ attains the same value for some mutually distinct $x_{1}$ and $x_{2}$, providing that $x_{1}<X_{0}$ and $X_{0}<x_{2}$. Furthermore, the identity $W_{\mathrm{T}}^{\text {aux }}(x)=W_{\mathrm{T}}^{\text {aux }}\left(2 X_{0}-x\right)$ (see (73)) implies that $x_{2}=2 X_{0}-x_{1}$. Consequently, (39) is the only solution of (72).

\section{Substitution of (39) into (66)-(67) yields (40)-(41).}

Taking into account (39), the right-hand side of (70) can be expressed in terms of $x_{\max }$ only,

$$
C_{0} U_{\max }=\operatorname{artanh} \frac{x_{\max }-X_{0}-b_{1}}{B_{0}}-\operatorname{artanh} \frac{X_{0}-x_{\max }-b_{1}}{B_{0}} .
$$

Applying $\tanh (\cdot)$ to the both sides of (75) and using the identity

$$
\tanh (u+v)=\frac{\tanh (u)+\tanh (v)}{1+\tanh (u) \tanh (v)}
$$

we obtain

$$
\tanh \left(C_{0} U_{\max }\right)=\frac{2 B_{0}\left(x_{\max }-X_{0}\right)}{\left(x_{\max }-X_{0}\right)^{2}+B_{0}^{2}-b_{1}^{2}} .
$$

Equation (77) is quadratic in terms of $\left(x_{\max }-X_{0}\right)$ :

$$
\alpha\left(x_{\max }-X_{0}\right)^{2}-2 B_{0}\left(x_{\max }-X_{0}\right)+\left(B_{0}^{2}-b_{1}^{2}\right) \alpha=0
$$

where $\alpha=\tanh \left(C_{0} U_{\max }\right)$. Notice that $U_{\max }>0$ implies that $0<\alpha<1$, which further implies that $B_{0}{ }^{2}\left(1-\alpha^{2}\right)+b_{1}{ }^{2} \alpha^{2}$ is positive. Thus, (78) has two real solutions, one of which is given by (42). Since the other solution contradicts (34), it follows that (42) is the only acceptable solution.

\section{Acknowledgments}

The authors would like to thank Professor Ladislav A. Novak for his valuable suggestions and comments in the course of development of this work.

This work was supported in part by the European Union's Horizon 2020 Research and Innovation Programme under the Marie Sklodowska-Curie Grant Agreement 734331, and in part by the Serbian Ministry of Education, Science and Technology Development under Grant TR32016 and Grant III45021.

We are grateful to the authors, publisher of the journal and RightsLink service for permissions to reprint figures from the paper [54]. 


\section{References}

[1] TAN, X., IYER, R. V. Modeling and control of hysteresis. IEEE Control Systems Magazine, 2009, vol. 29, no. 1, p. 26-28. DOI: 10.1109/mcs.2008.930921

[2] SMITH, R. C. Smart Material Systems: Model Development. Philadelphia, PA (USA): SIAM, 2005. ISBN: 9780898715835

[3] KRASNOSEL'SKII, M. A., POKROVSKII, A. V. Systems with Hysteresis. Berlin (Germany): Springer-Verlag, 1989. ISBN: 9783642613029

[4] MAYERGOYZ, I. D. Mathematical Models of Hysteresis. Berlin (Germany): Springer-Verlag, 1991. ISBN: 9781461230281

[5] VISINTIN, A. Differential Models of Hysteresis. Berlin (Germany): Springer-Verlag, 1994. ISBN: 9783662115572

[6] BROKATE, M., SPREKELS, J. Hysteresis and Phase Transitions. Berlin (Germany): Springer-Verlag, 1996. ISBN: 9781461240488

[7] IVÁNYI, A. Hysteresis Models in Electromagnetic Computation. Budapest (Hungary): ISBSI, 1997. ISBN: 9630574160

[8] HADJIPANAYIS. G. C. (Ed.). Magnetic Hysteresis in Novel Magnetic Materials. Dordrecht (Netherlands): Springer Netherlands, 1997. ISBN: 9789401154789

[9] BERTOTTI, G. Hysteresis in Magnetism. Boston (USA): Academic Press, 1998. ISBN: 9780080534374

[10] DELlA TORRE, E. Magnetic Hysteresis. New York (USA): Wiley-IEEE Press, 1999. ISBN: 9780780347199

[11] TAKACS, J. Mathematics of Hysteretic Phenomena. Weinheim (Germany): Wiley-VCH, 2006. ISBN: 9783527404018

[12] DIMIAN, M., ANDREI, P. Noise-Driven Phenomena in Hysteretic Systems. New York (USA): Springer, 2014. ISBN: 9781461413745

[13] TEAPE, J. W., SIMPSON, R. R. S., SLATER, R. D., et al. Representation of magnetic characteristic, including hysteresis, by exponential series. Proceedings of the IEEE, 1974, vol. 121, no. 1, p. 1019-1020. DOI: 10.1049/piee.1974.0235

[14] OSSART, F., MEUNIER, G. Comparison between various hysteresis models and experimental data. IEEE Transactions on Magnetics, 1990, vol. 26, no. 5, p. 2837-2839. DOI: $10.1109 / 20.104893$

[15] DE LEON, F., SEMLYEN, A. A simple representation of dynamic hysteresis losses in power transformers. IEEE Transactions on Power Delivery, 1995, vol. 10, no. 1, p. 315-321. DOI: $10.1109 / 61.368383$

[16] WIDGER, G. F. T. Representation of magnetisation curves over extensive range by rational fraction approximations. Proceedings of the IEEE, 1969, vol. 116, no. 1, p. 156-160. DOI: 10.1049 /piee. 1969.0032

[17] RIVAS, J., ZAMARRO, J. M., MARTIN, E., et al Simple approximation for magnetization curves and hysteresis loops. IEEE Transactions on Magnetics, 1981, vol. 17, no. 4, p. 1498-1502. DOI: 10.1109/TMAG.1981.1061241

[18] BATTISTELli, L., GENTILE, G., PICCOLO, A. Representation of hysteresis loops by rational fraction approximations. Physica Scripta, 1989, vol. 40, no. 4, p. 502-507. DOI: 10.1088/0031$8949 / 40 / 4 / 012$

[19] SYKULSKI, J. K. Computational Magnetics. Dordrecht (Netherlands): Springer Netherlands, 1995. ISBN: 9789401112789

[20] SAITO, Y., HAYANO, S., NAKAMURA, H., et al. A representation of magnetic hysteresis by Fourier series. Journal of Magnetism and Magnetic Materials, 1986, vol. 54-57, Part 3, p. 1613-1614. DOI: 10.1016/0304-8853(86)90947-9
[21] WLODARSKI, Z., WLODARSKA, J., BRYKALSKI, A. Application of different saturation curves in a mathematical model of hysteresis. COMPEL - The International Journal for Computation and Mathematics in Electrical and Electronic Engineering, 2005, vol. 24, no. 4, p. 1367-1380. DOI: $10.1108 / 03321640510615661$

[22] WLODARSKI, Z. Modeling hysteresis by analytical reversal curves. Physica B: Condensed Matter, 2007, vol. 398, no. 1, p. 159-163. DOI: 10.1016/j.physb.2007.05.012

[23] AVANAKI, Z. A., HASSANZADEH, A. J. Modified Brillouin function to explain the ferromagnetic behavior of surfactant-aided synthesized $\alpha-\mathrm{Fe}_{2} \mathrm{O}_{3}$ nanostructures. Journal of Theoretical and Applied Physics, 2013, vol. 7, no. 19, p. 1-7. DOI: 10.1186/22517235-7-19

[24] POTTER, R. I., SCHMULIAN, R. J. Self-consistently computed magnetization patterns in thin magnetic recording media. IEEE Transactions on Magnetics, 1971, vol. 7, no. 4, p. 873-880. DOI: 10.1109/TMAG.1971.1067251

[25] TRUjILlo, H., CRUZ, J., RIVERO, M., et al. Analysis of the fluxgate response through a simple spice model. Sensors and Actuators A: Physical, 1999, vol. 75, no. 1, p. 1-7. DOI: 10.1016/S0924-4247(98)00280-5

[26] WANG, Y., WU, S., ZHOU, Z., et al. Research on the dynamic hysteresis loop model of the residence times difference (RTD)fluxgate. Sensors, 2013, vol. 13, no. 9, p. 11539-11552. DOI: $10.3390 / \mathrm{s} 130911539$

[27] MILOVANOVIC, A. M., KOPRIVICA, B. M. Mathematical model of major hysteresis loop and transient magnetizations. Electromagnetics, 2015, vol. 35, no. 3, p. 155-166. DOI: 10.1080/02726343.2015.1005202

[28] KOPRIVICA, B., MILOVANOVIC, A., MITROVIC, N. Mathematical modeling of frequency-dependent hysteresis and energy loss of FeBSiC amorphous alloy. Journal of Magnetism and Magnetic Materials, 2017, vol. 422, p. 37-42. DOI: 10.1016/j.jmmm.2016.08.061

[29] TAKACS, J. A phenomenological mathematical model of hysteresis. COMPEL - The International Journal for Computation and Mathematics in Electrical and Electronic Engineering, 2001, vol. 20, no. 4, p. 1002-1014. DOI: 10.1108/EUM0000000005771

[30] PETRESCU, L., CAZACU, E., PETRESCU, C. Sigmoid functions used in hysteresis phenomenon modeling. In 9th International Symposium on Advanced Topics in Electrical Engineering (ATEE). Bucharest (Romania), 2015, p. 521-524. DOI: 10.1109/ATEE.2015.7133863

[31] ADHIKARI, S. P., SAH, M. P., KIM, H., CHUA, L. O. Three fingerprints of memristor. IEEE Transactions on Circuits and Systems I: Regular Papers, 2013, vol. 60, no. 11, p. 3008-3021. DOI: 10.1109/TCSI.2013.2256171

[32] CHUA, L. O. If it's pinched it's a memristor. Semiconductor Science and Technology, 2014, vol. 29, no. 10, p. 1-42. DOI: 10.1088/0268-1242/29/10/104001

[33] CHUA, L. O. Everything you wish to know about memristors but are afraid to ask. Radioengineering, 2015, vol. 24, no. 2, p. 319-368. DOI: $10.13164 /$ re.2015.0319

[34] BIOLEK, D., BIOLEK, Z. About fingerprints of Chua's memristors. IEEE Circuits and Systems Magazine, 2018, vol. 18, no. 2, p. 35-47. DOI: 10.1109/MCAS.2018.2821759

[35] BIOLEK, D., BIOLEK, Z., BIOLKOVA, V. Pinched hysteretic loops of ideal memristors, memcapacitors and meminductors must be 'self-crossing'. Electronics Letters, 2011, vol. 47, no. 25, p. 1385-1387. DOI: 10.1049/el.2011.2913

[36] BIOLEK, Z., BIOLEK, D. How can the hysteresis loop of the ideal memristor be pinched? IEEE Transactions on Circuits and Systems 
II: Express Briefs, 2014, vol. 61, no. 7, p. 491-495. DOI: 10.1109/TCSII.2014.2327303

[37] BIOLEK, Z., BIOLEK, D., BIOLKOVA, V., et al. Comments on pinched hysteresis loops of memristive elements. Radioengineering, 2015, vol. 24, no. 4, p. 962-967. DOI: 10.13164/re.2015.0962

[38] BIOLEK, Z., BIOLEK, D., BIOLKOVA, V., et al. Variation of a classical fingerprint of ideal memristor. International Journal of Circuit Theory and Applications, 2016, vol. 44, no. 5, p. 1202-1207. DOI: $10.1002 /$ cta.2121

[39] BIOLEK, D., BIOLEK, Z. BIOLKOVA, V. Every nonlinear element from Chua's table can generate pinched hysteresis loops: generalised homothety theorem. Electronics Letters, 2016, vol. 52, no. 21 , p. 1744-1746. DOI: $10.1049 /$ el.2016.2961

[40] BIOLEK, D., BIOLEK, Z., BIOLKOVA, V., et al. About v-i pinched hysteresis of some non-memristive systems. Mathematical Problems in Engineering, 2018, vol. 2018, p. 1-10. DOI: $10.1155 / 2018 / 1747865$

[41] BIOLEK, D., BIOLEK, Z., BIOLKOVA, V. Interpreting area of pinched memristor hysteresis loop. Electronics Letters, 2014, vol. 50, no. 2, p. 74-75. DOI: 10.1049/el.2013.3108

[42] SAH, M. P., KIM, H., CHUA, L. O. Brains are made of memristors. IEEE Circuits and Systems Magazine, 2014, vol. 14, no. 1, p. 12-36. DOI: 10.1109/MCAS.2013.2296414

[43] BIOLEK, Z., BIOLEK, D., BIOLKOVA, V. Computation of the area of memristor pinched hysteresis loop. IEEE Transactions on Circuits and Systems II: Express Briefs, 2012, vol. 59, no. 9, p. 607-611. DOI: 10.1109/TCSII.2012.2208670

[44] BIOLEK, Z., BIOLEK, D., BIOLKOVA, V. Analytical computation of the area of pinched hysteresis loops of ideal memelements. Radioengineering, 2013, vol. 22, no. 1, p. 132-135. ISSN: $1210-2512$

[45] JUHAS, A., DAUTOVIC, S. Computation of pinched hysteresis loop area from memristance-vs-state map. IEEE Transactions on Circuits and Systems II: Express Briefs, 2019, vol. 66, no. 4, p. 677-681. DOI: $10.1109 /$ TCSII.2018.2868384

[46] ELWAKIL, A. S., FOUDA, M. E., RADWAN, A. G. A simple model of double-loop hysteresis behavior in memristive elements. IEEE Transactions on Circuits and Systems II: Express Briefs, 2013, vol. 60 , no. 8, p. 487-491. DOI: 10.1109/TCSII.2013.2268376

[47] MAUNDY, B., ELWAKIL, A. S., PSYCHALINOS, C. Correlation between the theory of Lissajous figures and the generation of pinched hysteresis loops in nonlinear circuits. IEEE Transactions on Circuits and Systems I: Regular Papers, 2019, vol. 66, no. 7, p. 2606-2614. DOI: 10.1109/TCSI.2019.2900944

[48] WANG, X., RON HUI, S. Y. Graphical modelling of pinched hysteresis loops of memristors. IET Science, Measurement \& Technology, 2017, vol. 11, no. 1, p. 86-96. DOI: 10.1049/ietsmt.2016.0210

[49] MIRANDA, E. Compact model for the major and minor hysteretic $\mathrm{I}-\mathrm{V}$ loops in nonlinear memristive devices. IEEE Transactions on Nanotechnology, 2015, vol. 14 , no. 5, p. 787-789. DOI: 10.1109/TNANO.2015.2455235

[50] PATTERSON, A. G., SUNE, J., MIRANDA, E. Voltage-driven hysteresis model for resistive switching: SPICE modeling and circuit applications. IEEE Transactions on Computer-Aided Design of Integrated Circuits and Systems, 2017, vol. 36, no. 12, p. 2044-2051. DOI: 10.1109/TCAD.2017.2756561

[51] BAO, B., QIAN, H., XU, Q., et al. Coexisting behaviors of asymmetric attractors in hyperbolic-type memristor based Hopfield neural network. Frontiers in Computational Neuroscience, 2017, vol. 11 , no. 81 , p. 1-14. DOI: 10.3389 /fncom. 2017.00081

[52] ASCOLI, A., TETZLAFF, R., BIOLEK, Z., et al. The art of finding accurate memristor model solutions. IEEE Journal on Emerging and Selected Topics in Circuits and Systems, 2015, vol. 5, no. 2, p. 133-142. DOI: 10.1109/JETCAS.2015.2426493

[53] KRZYSTECZKO, P., REISS, G., THOMAS, A. Memristive switching of $\mathrm{MgO}$ based magnetic tunnel junctions. Applied Physics Letters, 2009, vol. 95, no. 11, p. 1-3. DOI: $10.1063 / 1.3224193$

[54] CHANThBouala, A., GARCIA, V., CHERIFI, R. O., et al. A ferroelectric memristor. Nature Materials, 2012, vol. 11, no. 10, p. 860-864. DOI: 10.1038/NMAT3415

[55] MIRANDA, E., JIMENEZ, D., SUNE, J. The quantum pointcontact memristor. IEEE Electron Device Letters, 2012, vol. 33, no. 10 , p. 1474-1476. DOI: 10.1109/LED.2012.2210185

[56] FUKAMI, S., ZHANG, C., DUTTAGUPTA, S., et al. Magnetization switching by spin-orbit torque in an antiferromagnet-ferromagnet bilayer system. Nature Materials, 2016, vol. 15, no. 5, p. 535-541. DOI: 10.1038/NMAT4566

[57] FUKAMI, S., OHNO, H. Perspective: Spintronic synapse for artificial neural network. Journal of Applied Physics, 2018, vol. 124 , no. 15 , p. $1-8$. DOI: $10.1063 / 1.5042317$

[58] DUENAS, S., CASTAN, H., GARCIA, H., et al. Study of the admittance hysteresis cycles in $\mathrm{TiN} / \mathrm{Ti} / \mathrm{HfO}_{2} / \mathrm{W}$-based RRAM devices. Microelectronic Engineering, 2017, vol. 178, p. 30-33. DOI: 10.1016/j.mee.2017.04.020

[59] CASTAN, H., DUENAS, S., GARCIA, H., et al. Analysis and control of the intermediate memory states of RRAM devices by means of admittance parameters. Journal of Applied Physics, 2018, vol. 124 , no. 15 , p. $1-8$. DOI: $10.1063 / 1.5024836$

[60] YAN, Z. B., LIU, J. M. Coexistence of high performance resistance and capacitance memory based on multilayered metaloxide structures. Scientific Reports, 2013, vol. 3, p. 1-7. DOI: $10.1038 /$ srep02482

[61] BESSONOV, A. A., KIRIKOVA, M. N., PETUKHOV, D. I., et al. Layered memristive and memcapacitive switches for printable electronics. Nature Materials, 2015, vol. 14, no. 2, p. 199-204. DOI: 10.1038/NMAT4135

[62] LIN, D., RON HUI, S. Y., CHUA, L. O. Gas discharge lamps are volatile memristors. IEEE Transactions on Circuits and Systems I: Regular Papers, 2014, vol. 61, no. 7, p. 2066-2073. DOI: 10.1109/TCSI.2014.2304659

[63] BIOLEK, Z., BIOLEK, D., BIOLKOVA, V. SPICE model of memristor with nonlinear dopant drift. Radioengineering, 2009, vol. 18 , no. 2, p. 210-214. ISSN: 1210-2512

[64] PARKER, T. S., CHUA, L. O. Practical Numerical Algorithms for Chaotic Systems. New York (USA): Springer-Verlag, 1989. ISBN: 9781461234869

[65] SLIPKO, V. A., PERSHIN, Y. V. Transient dynamics of pulsedriven memristors in the presence of a stable fixed point. Physica E: Low-dimensional Systems and Nanostructures, 2019, vol. 114, p. 1-5. DOI: 10.1016/j.physe.2019.113561

[66] SLIPKO, V. A., PERSHIN, Y. V. Importance of the window function choice for the predictive modelling of memristors. IEEE Transactions on Circuits and Systems II: Express Briefs, 2019, early access, p. 1-5. DOI: 10.1109/TCSII.2019.2906295

[67] CORINTO, F., ASCOLI, A. A boundary condition-based approach to the modeling of memristor nano-structures. IEEE Transactions on Circuits and Systems-I: Regular Papers, 2012, vol. 59, no. 11, p. 2713-2726. DOI: 10.1109/TCSI.2012.2190563 


\section{About the Authors ...}

Stanisa DAUTOVIC obtained the B.Sc. degree in Electrical Engineering in 1991 from the Univ. of Novi Sad, Dept. of Electrical Engineering and Computing; the M.Sc. degree in Discrete Mathematics and Computer Science in 1997 from the Univ. of Novi Sad, Faculty of Science and Mathematics; and the Ph.D. degree in Electrical Engineering in 2009 from the Univ. of Novi Sad, Dept. of Electrical Engineering and Computing. He joined the Dept. of Electrical Engineering and Computing at Univ. of Novi Sad in 1991, where he is currently an Assistant Professor. His research interest includes circuits, signals and systems, algorithms and computational complexity.

Natasa SAMARDZIC (corresponding author) obtained the B.Sc., M.Sc. and Ph.D. degrees in Electrical Engineer- ing from the Faculty of Technical Sciences, University of Novi Sad, in 2009, 2010 and 2016, respectively. She is currently an Assistant Professor with the Department of Electronics, Faculty of Technical Sciences, University of Novi Sad. Her research interest includes modeling and fabrication of nanoelectronic devices and characterization of nanomaterials.

Anamarija JUHAS obtained her Ph.D. degree in Electrical Engineering from the Faculty of Technical Sciences, University of Novi Sad, Serbia, in 2009. She is currently an Associate Professor with the Department of Power, Electronic and Telecommunication Engineering at the University of Novi Sad. Her research interests include memristors, power amplifiers and electromagnetic compatibility. 